\title{
Renormalization Group Reduction of Non Integrable Hamiltonian Systems
}

\author{
Stephan I. Tzenov \\ Plasma Physics Laboratory, Princeton University, Princeton, New Jersey 08543
}

\begin{abstract}
Based on the Renormalization Group method, a reduction of non integrable multi-dimensional hamiltonian systems has been performed. The evolution equations for the slowly varying part of the angle-averaged phase space density, and for the amplitudes of the angular modes have been derived. It has been shown that these equations are precisely the Renormalization Group equations. As an application of the approach developed, the modulational diffusion in one-and-ahalf degree of freedom dynamical system has been studied in detail.
\end{abstract}




\section{Introduction}

It is well-known that dynamical systems may exhibit irregular motion in certain regions of phase spacel1. These regions differ in size, from being considerably small, to occupying large parts of phase space. This depends mostly on the strength of the perturbation, as well as on the intrinsic characteristics of the system. For comparatively small perturbations the regularity of the motion is expressed in the existence of adiabatic action invariants. In the course of nonlinear interaction the action invariants vary within a certain range, prescribed by the integrals of motion (if such exist). For chaotic systems some (or all) of the integrals of motion are destroyed, causing specific trajectories to become extremely complicated. These trajectories look random in their behavior, therefore it is natural to explore the statistical properties of chaotic dynamical systems.

Much experimental and theoretical evidence th of nonlinear effects in the dynamics of particles in accelerators and storage rings is available at present. An individual particle propagating in an accelerator experiences growth of amplitude of betatron oscillations in a plane transverse to the particle orbit, whenever a perturbing force acts on it. This force may be of various origin, for instance high order multipole magnetic field errors, space charge forces, beam-beam interaction force, power supply ripple or other external and collective forces. Therefore, the Hamiltonian governing the motion of a beam particle is far from being integrable, and an irregular behavior of the beam is clearly observed, especially for a large number of turns.

The idea to treat the evolution of chaotic dynamical systems in a statistical sense is not new; many rigorous results related to the statistical properties of such systems can be found in the book by Arnold and Avez5. Many of the details concerning the transport phenomena in the space of adiabatic action invariants only are also well understoode. In this aspect the results presented here are in a sense re-derivation of previously obtained ones by means of a different method. What is new however, is the approach followed to obtain the diffusion properties in action variable space, as well as a new evolution equation for the angle-dependent part of the phase space density. Furthermore, instead of the widely used phenomenological method to derive the diffusion coefficient (tensor), the procedure pursued in the present paper is more consistent one, with a starting point the Liouville's equation for the phase space density.

We first employ the Projection Operator method of Zwanzig6 to derive the equations for the two parts of the phase space density: the averaged over the angle variables part $F$, and the remainder $G$ [see Eq. (2.10) in the next Section]. As expected, the two equations are coupled. Next we extract the relevant long-time scale behavior embedded in the equations for $F$ and $G$ by means of the Renormalization Group (RG) method 6 . It is remarkable, and at the same time not surprising that the equations governing the long-time scale dynamics are the Renormalization Group equations (RGEs). These are obtained in Section 4 through renormalizing the perturbative solution of the equations for $F$ and $G$ [see Eqs. (2.22) and (2.23) of Section 2]. Finally, in Section 5 one-dimensional example of a chaotic system is considered to demonstrate the approach developed here. 


\section{Projection Operator Method}

Single particle dynamics in cyclic accelerators and storage rings is most properly described by the adiabatic action invariants (Courant-Snyder invariants 9 ) and the canonically conjugate to them angle variables. However, to be more general we consider here a dynamical system with $N$ degrees of freedom, governed by the Hamiltonian written in action-angle variables $(\boldsymbol{J}, \boldsymbol{\alpha})$ as

$H(\boldsymbol{\alpha}, \boldsymbol{J} ; \theta)=H_{0}(\boldsymbol{J})+\epsilon V(\boldsymbol{\alpha}, \boldsymbol{J} ; \theta)$,

where $\theta$ is the independent azimuthal variable (widely used in accelerator physics), playing the role of time and $\boldsymbol{J}$ and $\boldsymbol{\alpha}$ are $N$-dimensional vectors

$\boldsymbol{J}=\left(J_{1}, J_{2}, \ldots, J_{N}\right), \quad \boldsymbol{\alpha}=\left(\alpha_{1}, \alpha_{2}, \ldots, \alpha_{N}\right)$

Moreover $H_{0}(\boldsymbol{J})$ is the integrable part of the Hamiltonian, $\epsilon$ is a formal small parameter, while $V(\boldsymbol{\alpha}, \boldsymbol{J} ; \theta)$ is the perturbation periodic in the angle variables

$V(\boldsymbol{\alpha}, \boldsymbol{J} ; \theta)=\sum_{\boldsymbol{m}}^{\prime} V_{\boldsymbol{m}}(\boldsymbol{J} ; \theta) \exp (i \boldsymbol{m} \cdot \boldsymbol{\alpha})$,

where $\sum^{\prime}$ denotes exclusion of the harmonic $\boldsymbol{m}=(0,0, \ldots, 0)$ from the above sum. The Hamilton's equations of motion are

$\frac{\mathrm{d} \alpha_{k}}{\mathrm{~d} \theta}=\omega_{0 k}(\boldsymbol{J})+\epsilon \frac{\partial V}{\partial J_{k}}, \quad \frac{\mathrm{d} J_{k}}{\mathrm{~d} \theta}=-\epsilon \frac{\partial V}{\partial \alpha_{k}}$

where

$\omega_{0 k}(\boldsymbol{J})=\frac{\partial H_{0}}{\partial J_{k}}$

In what follows (in particular in Section 4) we assume that the nonlinearity coefficients

$\gamma_{k l}(\boldsymbol{J})=\frac{\partial^{2} H_{0}}{\partial J_{k} \partial J_{l}}$

are small and can be neglected. The Liouville's equation governing the evolution of the phase space density $P(\boldsymbol{\alpha}, \boldsymbol{J} ; \theta)$ can be written as

$\frac{\partial}{\partial \theta} P(\boldsymbol{\alpha}, \boldsymbol{J} ; \theta)=\left[\widehat{\mathcal{L}}_{0}+\epsilon \widehat{\mathcal{L}}_{v}(\theta)\right] P(\boldsymbol{\alpha}, \boldsymbol{J} ; \theta)$.

Here the operators $\widehat{\mathcal{L}}_{0}$ and $\widehat{\mathcal{L}}_{v}$ are given by the expressions

$\widehat{\mathcal{L}}_{0}=-\omega_{0 k}(\boldsymbol{J}) \frac{\partial}{\partial \alpha_{k}}, \quad \widehat{\mathcal{L}}_{v}=\frac{\partial V}{\partial \alpha_{k}} \frac{\partial}{\partial J_{k}}-\frac{\partial V}{\partial J_{k}} \frac{\partial}{\partial \alpha_{k}}$

where summation over repeated indices is implied. Next we define the projection operator onto the subspace of action variables by the following integral: 
$\widehat{\mathcal{P}} f(\boldsymbol{J} ; \theta)=\frac{1}{(2 \pi)^{N}} \int_{0}^{2 \pi} \mathrm{d} \alpha_{1} \ldots \int_{0}^{2 \pi} \mathrm{d} \alpha_{N} f(\boldsymbol{\alpha}, \boldsymbol{J} ; \theta)$,

where $f(\boldsymbol{\alpha}, \boldsymbol{J} ; \theta)$ is a generic function of its arguments. Let us introduce also the functions $F=\widehat{\mathcal{P}} P$

$$
G=(1-\widehat{\mathcal{P}}) P=\widehat{\mathcal{C}} P
$$$$
(P=F+G)
$$

From Eq. (2.7) with the obvious relations

$\widehat{\mathcal{P}} \widehat{\mathcal{L}}_{0}=\widehat{\mathcal{L}}_{0} \widehat{\mathcal{P}} \equiv 0, \quad \widehat{\mathcal{P}} \widehat{\mathcal{L}}_{v} \widehat{\mathcal{P}} \equiv 0$

in hand it is straightforward to obtain the equations

$\frac{\partial F}{\partial \theta}=\epsilon \widehat{\mathcal{P}} \widehat{\mathcal{L}}_{v} G=\epsilon \frac{\partial}{\partial J_{k}} \widehat{\mathcal{P}}\left(\frac{\partial V}{\partial \alpha_{k}} G\right)$

$\frac{\partial G}{\partial \theta}=\widehat{\mathcal{L}}_{0} G+\epsilon \widehat{\mathcal{C}} \widehat{\mathcal{L}}_{v} G+\epsilon \widehat{\mathcal{L}}_{v} F$

Our goal in the subsequent exposition is to analyze Eqs. (2.12) and (2.13) using the RG method. It will prove efficient to eliminate the dependence on the angle variables in $G$ and $V$ by noting that the eigenfunctions of the operator $\widehat{\mathcal{L}}_{0}$ form a complete set, so that every function periodic in the angle variables can be expanded in this basis. Using Dirac's "bra-ket" notation we write

$|\boldsymbol{n}\rangle=\frac{1}{(2 \pi)^{N / 2}} \exp (i \boldsymbol{n} \cdot \boldsymbol{\alpha}), \quad\langle\boldsymbol{n}|=\frac{1}{(2 \pi)^{N / 2}} \exp (-i \boldsymbol{n} \cdot \boldsymbol{\alpha})$.

The projection operator $\widehat{\mathcal{P}}$ can be represented in the form 10

$\widehat{\mathcal{P}}=\widehat{\mathcal{P}}_{\mathbf{0}}=|\mathbf{0}\rangle\langle\mathbf{0}|$.

One can also define a set of projection operators $\widehat{\mathcal{P}}_{\boldsymbol{n}}$ according to the expression 10

$\widehat{\mathcal{P}}_{\boldsymbol{n}}=|\boldsymbol{n}\rangle\langle\boldsymbol{n}|$.

It is easy to check the completeness relation

$$
\sum_{n} \widehat{\mathcal{P}}_{n}=1
$$

from which and from Eq. (2.15) it follows that

$\widehat{\mathcal{C}}=\sum_{n \neq 0}^{\prime}|\boldsymbol{n}\rangle\langle\boldsymbol{n}|$

Decomposing the quantities $F, G$ and $V$ in the basis (2.14) as

$F=F(\boldsymbol{J} ; \theta)|\mathbf{0}\rangle$, 
$G(\boldsymbol{\alpha}, \boldsymbol{J} ; \theta)=\sum_{\boldsymbol{m} \neq \mathbf{0}}^{\prime} G_{\boldsymbol{m}}(\boldsymbol{J} ; \theta)|\boldsymbol{m}\rangle$,

$V(\boldsymbol{\alpha}, \boldsymbol{J} ; \theta)=\sum_{\boldsymbol{n} \neq \mathbf{0}}^{\prime} V_{\boldsymbol{n}}(\boldsymbol{J} ; \theta)|\boldsymbol{n}\rangle$,

from Eqs. (2.12) and (2.13) we obtain

$\frac{\partial F}{\partial \theta}=i \epsilon \frac{\partial}{\partial J_{k}}\left(\sum_{n}^{\prime} n_{k} V_{\boldsymbol{n}} G_{-n}\right)$,

$\frac{\partial G_{\boldsymbol{n}}}{\partial \theta}=-i n_{k} \omega_{0 k} G_{\boldsymbol{n}}+i \epsilon \sum_{\boldsymbol{m}}^{\prime}\left[n_{k} V_{\boldsymbol{n}-\boldsymbol{m}} \frac{\partial G_{\boldsymbol{m}}}{\partial J_{k}}-m_{k} \frac{\partial}{\partial J_{k}}\left(V_{\boldsymbol{n}-\boldsymbol{m}} G_{\boldsymbol{m}}\right)\right]+i \epsilon n_{k} V_{\boldsymbol{n}} \frac{\partial F}{\partial J_{k}}$.

The above equations comprise the the starting point in the analysis outlined in Section 4. We are primarily interested in the long-time evolution of the original system governed by certain amplitude equations. These will turn out to be precisely the RG equations.

\section{Renormalization Group Reduction of Hamilton's Equations}

Let us consider the solution of Hamilton's equations of motion (2.4) for small perturbation parameter $\epsilon$. It is natural to introduce the perturbation expansion

$\alpha_{k}=\alpha_{k}^{(0)}+\epsilon \alpha_{k}^{(1)}+\epsilon^{2} \alpha_{k}^{(2)}+\cdots, \quad \quad J_{k}=J_{k}^{(0)}+\epsilon J_{k}^{(1)}+\epsilon^{2} J_{k}^{(2)}+\cdots$.

The lowest order perturbation equations have the trivial solution:

$\alpha_{k}^{(0)}=\omega_{0 k} \theta+\varphi_{k}, \quad J_{k}^{(0)}=A_{k}$,

where $A_{k}$ and $\varphi_{k}$ are constant amplitude and phase, respectively. We write the first order perturbation equations as

$\frac{\mathrm{d} \alpha_{k}^{(1)}}{\mathrm{d} \theta}=\gamma_{k l}(\boldsymbol{A}) J_{l}^{(1)}+\frac{\partial V}{\partial A_{k}}, \quad \frac{\mathrm{d} J_{k}^{(1)}}{\mathrm{d} \theta}=-\frac{\partial V}{\partial \alpha_{k}^{(0)}}$,

Assuming that the modes $V_{\boldsymbol{n}}(\boldsymbol{J} ; \theta)$ are periodic in $\theta$, we can expand them in a Fourier series

$V_{\boldsymbol{n}}(\boldsymbol{J} ; \theta)=\sum_{\mu=-\infty}^{\infty} V_{\boldsymbol{n}}(\boldsymbol{J} ; \mu) \exp \left(i \mu \nu_{\boldsymbol{n}} \theta\right)$

If the original system (2.1) is far from primary resonances of the form:

$n_{k}^{(R)} \omega_{0 k}+\mu \nu_{R}=0$

we can solve the first order perturbation equations (3.3), yielding the result: 


$$
\begin{aligned}
\alpha_{k}^{(1)} & =i \sum_{\boldsymbol{m}}^{\prime} \sum_{\mu} \gamma_{k l}(\boldsymbol{A}) m_{l} V_{\boldsymbol{m}}(\mu) \frac{\exp \left[i\left(m_{s} \omega_{0 s}+\mu \nu_{\boldsymbol{m}}\right) \theta\right]}{\left(m_{s} \omega_{0 s}+\mu \nu_{\boldsymbol{m}}\right)^{2}} \exp \left(i m_{s} \varphi_{s}\right) \\
& -i \sum_{\boldsymbol{m}}^{\prime} \sum_{\mu} \frac{\partial V_{\boldsymbol{m}}(\mu)}{\partial A_{k}} \frac{\exp \left[i\left(m_{s} \omega_{0 s}+\mu \nu_{\boldsymbol{m}}\right) \theta\right]}{m_{s} \omega_{0 s}+\mu \nu_{\boldsymbol{m}}} \exp \left(i m_{s} \varphi_{s}\right), \\
J_{k}^{(1)} & =-\sum_{\boldsymbol{m}}^{\prime} \sum_{\mu} m_{k} V_{\boldsymbol{m}}(\mu) \frac{\exp \left[i\left(m_{s} \omega_{0 s}+\mu \nu_{\boldsymbol{m}}\right) \theta\right]}{m_{s} \omega_{0 s}+\mu \nu_{\boldsymbol{m}}} \exp \left(i m_{s} \varphi_{s}\right),
\end{aligned}
$$

The second order perturbation equations have the form:

$$
\begin{aligned}
& \frac{\mathrm{d} \alpha_{k}^{(2)}}{\mathrm{d} \theta}=\gamma_{k l}(\boldsymbol{A}) J_{l}^{(2)}+\frac{1}{2} \frac{\partial \gamma_{k l}}{\partial A_{s}} J_{l}^{(1)} J_{s}^{(1)}+\frac{\partial^{2} V}{\partial A_{k} \partial A_{l}} J_{l}^{(1)}+\frac{\partial^{2} V}{\partial A_{k} \partial \alpha_{l}^{(0)}} \alpha_{l}^{(1)}, \\
& \frac{\mathrm{d} J_{k}^{(2)}}{\mathrm{d} \theta}=-\frac{\partial^{2} V}{\partial \alpha_{k}^{(0)} \partial A_{l}} J_{l}^{(1)}-\frac{\partial^{2} V}{\partial \alpha_{k}^{(0)} \partial \alpha_{l}^{(0)}} \alpha_{l}^{(1)} .
\end{aligned}
$$

The solution to Eq. (3.9) reads as

$$
\begin{aligned}
J_{k}^{(2)} & =2 \pi \mathcal{R}(\theta) \sum_{\boldsymbol{m}>\mathbf{0}}^{\prime} \sum_{\mu} m_{k} m_{l} \frac{\partial\left|V_{\boldsymbol{m}}(\mu)\right|^{2}}{\partial A_{l}} \Re\left(\gamma ; m_{s} \omega_{0 s}+\mu \nu_{\boldsymbol{m}}\right) \\
& +\left.2 \pi \mathcal{R}(\theta) \sum_{\boldsymbol{m}>\mathbf{0}}^{\prime} \sum_{\mu} m_{k} m_{l} m_{s} \gamma_{l s}(\boldsymbol{A})\left|V_{\boldsymbol{m}}(\mu)\right|^{\mathbf{2}} \frac{\partial}{\partial a} \Re(\gamma ; a)\right|_{a=m_{r} \omega_{0 r}+\mu \nu_{\boldsymbol{m}}} \\
& + \text { oscillating terms, }
\end{aligned}
$$

where

$\frac{\mathrm{d} \mathcal{R}}{\mathrm{d} \theta}=1$

$\pi \Re(x ; y)=\frac{x}{x^{2}+y^{2}}, \quad \quad \lim _{x \rightarrow 0} \Re(x ; y)=\delta(y)$,

and $\gamma$ is a small real quantity added $a d$ hoc in the denominators of the expressions (3.6) and (3.7). The limit $\gamma \rightarrow 0$ will be taken in the final result.

As expected, in the second order perturbation solution (3.10) the first and the second terms are secular, because $\mathcal{R}(\theta)=\theta$. To remove these secularities we follow the general prescription of the RG method 8 . First, we select the slowly varying part of the perturbation solution governing the long-time evolution of the system. Up to second order in the perturbation parameter $\epsilon$ it consists of the constant zero order term $A_{k}$ and the second order secular terms. Next, we introduce the intermediate time $\tau$, and in order to absorb the difference $\tau=\theta-(\theta-\tau)$ we make the new renormalized amplitude $A_{k}(\tau)$ dependent on $\tau$. Since the long-time solution thus constructed should not depend on $\tau$ its derivative with respect to $\tau$ must be equal to zero. This also holds for $\tau=\theta$, so that finally 


$$
\begin{aligned}
\frac{\mathrm{d} A_{k}(\theta)}{\mathrm{d} \theta} & =2 \pi \epsilon^{2} \sum_{\boldsymbol{m}>\mathbf{0}}^{\prime} \sum_{\mu} m_{k} m_{l} \frac{\partial\left|V_{\boldsymbol{m}}(\boldsymbol{A} ; \mu)\right|^{\mathbf{2}}}{\partial A_{l}} \Re\left(\gamma ; m_{s} \omega_{0 s}+\mu \nu_{\boldsymbol{m}}\right) \\
& +\left.2 \pi \epsilon^{2} \sum_{\boldsymbol{m}>\mathbf{0}}^{\prime} \sum_{\mu} m_{k} m_{l} m_{s} \gamma_{l s}(\boldsymbol{A})\left|V_{\boldsymbol{m}}(\boldsymbol{A} ; \mu)\right|^{\mathbf{2}} \frac{\partial}{\partial a} \Re(\gamma ; a)\right|_{a=m_{r} \omega_{0 r}+\mu \nu_{\boldsymbol{m}}} .
\end{aligned}
$$

Equation (3.13) is known as the Renormalization Group equation (RGE). It describes the slow long-time evolution of the action variables.

\section{Renormalization Group Reduction of Liouville's Equation}

We consider the solution of Eqs. (2.22) and (2.23) for small $\epsilon$ by means of the RG method. For that purpose we perform again the naive perturbation expansion

$$
F=F^{(0)}+\epsilon F^{(1)}+\epsilon^{2} F^{(2)}+\cdots, \quad G_{\boldsymbol{n}}=G_{\boldsymbol{n}}^{(0)}+\epsilon G_{\boldsymbol{n}}^{(1)}+\epsilon^{2} G_{\boldsymbol{n}}^{(2)}+\cdots,
$$

and substitute it in Eqs. (2.22) and (2.23). The lowest order perturbation equations have the obvious solution

$$
F^{(0)}=F_{0}(\boldsymbol{J}), \quad G_{\boldsymbol{n}}^{(0)}=W_{\boldsymbol{n}}(\boldsymbol{J}) \exp \left(-i n_{k} \omega_{0 k} \theta\right)
$$

The first order perturbation equations read as:

$$
\begin{aligned}
& \frac{\partial F^{(1)}}{\partial \theta}=i \frac{\partial}{\partial J_{k}}\left[\sum_{\boldsymbol{n}}^{\prime} n_{k} V_{\boldsymbol{n}} W_{-\boldsymbol{n}} \exp \left(i n_{l} \omega_{0 l} \theta\right)\right] \\
& \frac{\partial G_{\boldsymbol{n}}^{(1)}}{\partial \theta}=-i n_{k} \omega_{0 k} G_{\boldsymbol{n}}^{(1)}+i n_{k} V_{\boldsymbol{n}} \frac{\partial F_{0}}{\partial J_{k}} \\
& +i \sum_{\boldsymbol{m}}^{\prime}\left[n_{k} V_{\boldsymbol{n}-\boldsymbol{m}} \frac{\partial W_{\boldsymbol{m}}}{\partial J_{k}}-m_{k} \frac{\partial}{\partial J_{k}}\left(V_{\boldsymbol{n}-\boldsymbol{m}} W_{\boldsymbol{m}}\right)\right] \exp \left(-i m_{l} \omega_{0 l} \theta\right) .
\end{aligned}
$$

We again assume that the modes $V_{\boldsymbol{n}}(\boldsymbol{J} ; \theta)$ are periodic in $\theta$, so that they can be expanded in a Fourier series (3.4). If the original system (2.1) exhibits primary resonances of the form (3.5) in the case when $\omega_{0 k}$ does not depend on the action variables, we can solve the first order perturbation equations (4.3) and (4.4). The result is as follows:

$$
\begin{aligned}
F^{(1)} & =i \mathcal{R}(\theta) \sum_{\boldsymbol{n}^{(R)}}^{\prime \prime} n_{k}^{(R)} \frac{\partial}{\partial J_{k}}\left[V_{\boldsymbol{n}^{(R)}}\left(-\frac{n_{l}^{(R)} \omega_{0 l}}{\nu_{R}}\right) W_{-\boldsymbol{n}^{(R)}}\right] \\
& +\sum_{\boldsymbol{n}}^{\prime} \sum_{\mu}^{\prime} n_{k} \frac{\partial}{\partial J_{k}}\left[V_{\boldsymbol{n}}(\mu) W_{-\boldsymbol{n}}\right] \frac{\exp \left[i\left(n_{l} \omega_{0 l}+\mu \nu_{\boldsymbol{n}}\right) \theta\right]}{n_{l} \omega_{0 l}+\mu \nu_{\boldsymbol{n}}}, \\
G_{\boldsymbol{n}}^{(1)} & =\mathcal{G}_{\boldsymbol{n}} \exp \left(-i n_{k} \omega_{0 k} \theta\right),
\end{aligned}
$$

where 


$$
\begin{aligned}
& \mathcal{G}_{\boldsymbol{n}}=i \mathcal{R}(\theta) \delta_{\boldsymbol{n} \boldsymbol{n}^{(R)}} n_{k} \frac{\partial F_{0}}{\partial J_{k}} V_{\boldsymbol{n}}\left(-\frac{n_{l}^{(R)} \omega_{0 l}}{\nu_{R}}\right) \\
& +i \mathcal{R}(\theta) \sum_{\boldsymbol{n}-\boldsymbol{n}^{(R)}}^{\prime \prime}\left\{n_{k} V_{\boldsymbol{n}^{(R)}}\left(-\frac{n_{l}^{(R)} \omega_{0 l}}{\nu_{R}}\right) \frac{\partial W_{\boldsymbol{n}-\boldsymbol{n}^{(R)}}}{\partial J_{k}}\right. \\
& \left.-\left(n_{k}-n_{k}^{(R)}\right) \frac{\partial}{\partial J_{k}}\left[V_{\boldsymbol{n}^{(R)}}\left(-\frac{n_{l}^{(R)} \omega_{0 l}}{\nu_{R}}\right) W_{\left.\boldsymbol{n}-\boldsymbol{n}^{(R)}\right)}\right]\right\} \\
& +n_{k} \frac{\partial F_{0}}{\partial J_{k}} \sum_{\mu}^{\prime} V_{\boldsymbol{n}}(\mu) \frac{\exp \left[i\left(n_{l} \omega_{0 l}+\mu \nu_{\boldsymbol{n}}\right) \theta\right]}{n_{l} \omega_{0 l}+\mu \nu_{\boldsymbol{n}}} \\
& +\sum_{\boldsymbol{m}}^{\prime} \sum_{\mu}^{\prime}\left\{n_{k} V_{\boldsymbol{n}-\boldsymbol{m}}(\mu) \frac{\partial W_{\boldsymbol{m}}}{\partial J_{k}}-m_{k} \frac{\partial}{\partial J_{k}}\left[V_{\boldsymbol{n}-\boldsymbol{m}}(\mu) W_{\boldsymbol{m}}\right]\right\} \frac{\exp \left\{i\left[\left(n_{l}-m_{l}\right) \omega_{0 l}+\mu \nu_{\boldsymbol{n}-\boldsymbol{m}}\right] \theta\right\}}{\left(n_{l}-m_{l}\right) \omega_{0 l}+\mu \nu_{\boldsymbol{n}-\boldsymbol{m}}}
\end{aligned}
$$

In the above expressions $\sum^{\prime \prime}$ denotes summation over all primary resonances (3.5). To obtain the desired RG equations we proceed in the same way as in the previous Section. The first order RG equations are

$$
\begin{aligned}
& \frac{\partial F_{0}}{\partial \theta}=i \epsilon \sum_{\boldsymbol{n}^{(R)}}^{\prime \prime} n_{k}^{(R)} \frac{\partial}{\partial J_{k}}\left[V_{\boldsymbol{n}^{(R)}}\left(\boldsymbol{J} ; \mu_{R}\right) W_{-\boldsymbol{n}^{(R)}}(\boldsymbol{J})\right], \\
& \frac{\partial W_{\boldsymbol{n}}}{\partial \theta}=i \epsilon \delta_{\boldsymbol{n} \boldsymbol{n}^{(R)}} n_{k} \frac{\partial F_{0}}{\partial J_{k}} V_{\boldsymbol{n}}\left(\boldsymbol{J} ; \mu_{R}\right) \\
& +i \epsilon \sum_{\boldsymbol{n}-\boldsymbol{n}^{(R)}} "\left\{n_{k} V_{\boldsymbol{n}^{(R)}}\left(\boldsymbol{J} ; \mu_{R}\right) \frac{\partial W_{\boldsymbol{n}-\boldsymbol{n}^{(R)}}}{\partial J_{k}}-\left(n_{k}-n_{k}^{(R)}\right) \frac{\partial}{\partial J_{k}}\left[V_{\boldsymbol{n}^{(R)}}\left(\boldsymbol{J} ; \mu_{R}\right) W_{\boldsymbol{n}-\boldsymbol{n}^{(R)}}(\boldsymbol{J})\right]\right\},
\end{aligned}
$$

where

$\mu_{R}=-\frac{n_{k}^{(R)} \omega_{0 k}}{\nu_{R}}$.

Equations (4.8) and (4.9) describe the resonant mode coupling when strong primary resonances are present in the original system.

Let us now assume that the original system is far from resonances. Solving the second order perturbation equation for $F^{(2)}$ and $G_{\boldsymbol{n}}^{(2)}$

$$
\frac{\partial F^{(2)}}{\partial \theta}=i \frac{\partial}{\partial J_{k}}\left(\sum_{n}^{\prime} n_{k} V_{\boldsymbol{n}} G_{-n}^{(1)}\right)
$$


$\frac{\partial G_{\boldsymbol{n}}^{(2)}}{\partial \theta}+i n_{k} \omega_{0 k} G_{\boldsymbol{n}}^{(2)}=i n_{k} V_{\boldsymbol{n}} \frac{\partial F^{(1)}}{\partial J_{k}}+i \sum_{\boldsymbol{m}}^{\prime}\left[n_{k} V_{\boldsymbol{n}-\boldsymbol{m}} \frac{\partial G_{\boldsymbol{m}}^{(1)}}{\partial J_{k}}-m_{k} \frac{\partial}{\partial J_{k}}\left(V_{\boldsymbol{n}-\boldsymbol{m}} G_{\boldsymbol{m}}^{(1)}\right)\right]$,

we obtain

$F^{(2)}=2 \pi \mathcal{R}(\theta) \frac{\partial}{\partial J_{k}}\left[\sum_{\boldsymbol{n}>0}^{\prime} \sum_{\lambda} n_{k} n_{l}\left|V_{\boldsymbol{n}}(\lambda)\right|^{2} \frac{\partial F_{0}}{\partial J_{l}} \Re\left(\gamma ; n_{s} \omega_{0 s}+\lambda \nu_{\boldsymbol{n}}\right)\right]+$ oscillating terms,

$G_{\boldsymbol{n}}^{(2)}=\mathcal{F}_{\boldsymbol{n}} \exp \left(-i n_{s} \omega_{0 s} \theta\right)$

where

$\mathcal{F}_{\boldsymbol{n}}=i \mathcal{R}(\theta) n_{k} n_{l} \sum_{\mu} \frac{V_{\boldsymbol{n}}(\mu)}{n_{s} \omega_{0 s}+\mu \nu_{\boldsymbol{n}}} \frac{\partial^{2}}{\partial J_{k} \partial J_{l}}\left[V_{\boldsymbol{n}}^{*}(\mu) W_{\boldsymbol{n}}\right]$

$+i \mathcal{R}(\theta) \sum_{\boldsymbol{m}}^{\prime} \sum_{\mu} \frac{1}{\left(n_{s}-m_{s}\right) \omega_{0 s}+\mu \nu_{\boldsymbol{n}-\boldsymbol{m}}}\left\{-n_{k} m_{l} V_{\boldsymbol{n}-\boldsymbol{m}}(\mu) \frac{\partial}{\partial J_{k}}\left(V_{\boldsymbol{n}-\boldsymbol{m}}^{*}(\mu) \frac{\partial W_{\boldsymbol{n}}}{\partial J_{l}}\right)\right.$

$+n_{k} n_{l} V_{\boldsymbol{n}-\boldsymbol{m}}(\mu) \frac{\partial^{2}}{\partial J_{k} \partial J_{l}}\left(V_{\boldsymbol{n}-\boldsymbol{m}}^{*}(\mu) W_{\boldsymbol{n}}\right)+m_{k} m_{l} \frac{\partial}{\partial J_{k}}\left(\left|V_{\boldsymbol{n}-\boldsymbol{m}}(\mu)\right|^{2} \frac{\partial W_{\boldsymbol{n}}}{\partial J_{l}}\right)$

$\left.-m_{k} n_{l} \frac{\partial}{\partial J_{k}}\left[V_{\boldsymbol{n}-\boldsymbol{m}}(\mu) \frac{\partial}{\partial J_{l}}\left(V_{\boldsymbol{n}-\boldsymbol{m}}^{*}(\mu) W_{\boldsymbol{n}}\right)\right]\right\}+$ oscillating terms

and the functions $\mathcal{R}(\theta)$ and $\Re(x ; y)$ are given by Eqs. (3.11) and (3.12), respectively. It is now straightforward to write the second order Renormalization Group equations. They are:

$$
\begin{aligned}
& \frac{\partial F_{0}}{\partial \theta}=2 \pi \epsilon^{2} \frac{\partial}{\partial J_{k}}\left[\sum_{\boldsymbol{n}>\mathbf{0}}^{\prime} \sum_{\lambda} n_{k} n_{l}\left|V_{\boldsymbol{n}}(\lambda)\right|^{\mathbf{2}} \frac{\partial F_{0}}{\partial J_{l}} \Re\left(\gamma ; n_{s} \omega_{0 s}+\lambda \nu_{\boldsymbol{n}}\right)\right], \\
& \frac{\partial W_{\boldsymbol{n}}}{\partial \theta}=i \epsilon^{2} n_{k} n_{l} \sum_{\mu} \frac{V_{\boldsymbol{n}}(\mu)}{n_{s} \omega_{0 s}+\mu \nu_{\boldsymbol{n}}} \frac{\partial^{2}}{\partial J_{k} \partial J_{l}}\left[V_{\boldsymbol{n}}^{*}(\mu) W_{\boldsymbol{n}}\right] \\
& +i \epsilon^{2} \sum_{\boldsymbol{m}}^{\prime} \sum_{\mu} \frac{1}{\left(n_{s}-m_{s}\right) \omega_{0 s}+\mu \nu_{\boldsymbol{n}-\boldsymbol{m}}}\left\{-n_{k} m_{l} V_{\boldsymbol{n}-\boldsymbol{m}}(\mu) \frac{\partial}{\partial J_{k}}\left(V_{\boldsymbol{n}-\boldsymbol{m}}^{*}(\mu) \frac{\partial W_{\boldsymbol{n}}}{\partial J_{l}}\right)\right. \\
& +n_{k} n_{l} V_{\boldsymbol{n}-\boldsymbol{m}}(\mu) \frac{\partial^{2}}{\partial J_{k} \partial J_{l}}\left(V_{\boldsymbol{n}-\boldsymbol{m}}^{*}(\mu) W_{\boldsymbol{n}}\right)+m_{k} m_{l} \frac{\partial}{\partial J_{k}}\left(\left|V_{\boldsymbol{n}-\boldsymbol{m}}(\mu)\right|^{2} \frac{\partial W_{\boldsymbol{n}}}{\partial J_{l}}\right) \\
& \left.-m_{k} n_{l} \frac{\partial}{\partial J_{k}}\left[V_{\boldsymbol{n}-\boldsymbol{m}}(\mu) \frac{\partial}{\partial J_{l}}\left(V_{\boldsymbol{n}-\boldsymbol{m}}^{*}(\mu) W_{\boldsymbol{n}}\right)\right]\right\} .
\end{aligned}
$$


The RG equation (4.16) is a Fokker-Planck equation describing the diffusion of the adiabatic action invariant. It has been derived previously by many authors (see e.g. the book by Lichtenberg and Liebermant and the references therein). It is important to note that our derivation does not require the initial assumption concerning the fast stochastization of the angle variable. The fact that the latter is indeed a stochastic variable is clearly visible from the second RG equation (4.17), governing the slow amplitude evolution of the angle-dependent part of the phase space density. Nevertheless it looks complicated, its most important feature is that equations for the amplitudes of different modes are decoupled. In the case of isolated nonlinear resonance Eq. (4.17) acquires a very simple form as will be shown in the next Section.

\section{Modulational Diffusion}

As an example to demonstrate the theory developed in previous sections, we consider the simplest example of one-and-a-half degree of freedom dynamical system exhibiting chaotic motion

$$
H_{0}(J)=\lambda J+H_{s}(J), \quad V(\alpha, J ; \theta)=V(J) \cos (\alpha+\xi \sin \nu \theta) .
$$

The Hamiltonian (5.1), written in resonant canonical variables describes an isolated nonlinear resonance of one-dimensional betatron motion of particles in an accelerator with modulated resonant phase (or modulated linear betatron tune). The modulation may come from various sources: ripple in the power supply of quadrupole magnets, synchro-betatron coupling or ground motion. The resonance detuning $\lambda$ defines the distance from the resonance, $\Xi$ is the amplitude of modulation of the linear betatron tune and $\nu$ is the modulation frequency, where $\xi=\Xi / \nu$. Without loss of generality we consider $\xi$ positive. Since

$\omega_{0}=\lambda+\frac{\mathrm{d} H_{s}}{\mathrm{~d} J}, \quad V_{1}(J ; \mu)=\frac{1}{2} V(J) \mathcal{J}_{\mu}(\xi)$,

where $\mathcal{J}_{n}(z)$ is the Bessel function of order $n$, the RG equation (3.13) for the amplitude $A$ can be rewritten as

$$
\frac{\mathrm{d} A}{\mathrm{~d} \theta}=\frac{\pi \epsilon^{2}}{2 \nu}\left\{\frac{\partial}{\partial A}\left[V^{2}(A) \mathcal{J}_{\left[\frac{\omega_{0}}{\nu}\right]}^{2}(\xi)\right]-\left.\gamma(A) V^{2}(A) \frac{\partial}{\partial a}\left[\mathcal{J}_{a}^{2}(\xi)\right]\right|_{a=-\frac{\omega_{0}}{\nu}}\right\}
$$

Here the square brackets $[z]$ encountered in the index of the Bessel function imply integer part of $z$. Moreover, in deriving the expression for $V_{1}(J ; \mu)$ in Eq. (5.2) use has been made of the identity

$\exp (i q \sin z)==\sum_{n=-\infty}^{\infty} \mathcal{J}_{n}(|q|) \exp [i n z \operatorname{sgn}(q)]$

and finally, the limit $\gamma \rightarrow 0$ in Eq. (3.13) has been taken. For small value of $\xi$ utilizing the approximate expression for the derivative of Bessel functions with respect to the order we obtain 
$\frac{\mathrm{d} A}{\mathrm{~d} \theta}=\frac{\pi \epsilon^{2}}{2 \nu}\left\{\frac{\partial}{\partial A}\left[V^{2}(A) \mathcal{J}_{\left[\frac{\omega_{0}}{\nu}\right]}^{2}(\xi)\right]-2 \mathcal{J}_{\left[\frac{\omega_{0}}{\nu}\right]}^{2}(\xi) \ln \left(\frac{\xi}{2}\right) \gamma(A) V^{2}(A)\right\}$.

Let us now turn to the RG equations (4.16) and (4.17). They can be rewritten in the form:

$$
\begin{aligned}
& \frac{\partial F_{0}}{\partial \theta}=\frac{\pi \epsilon^{2}}{2 \nu} \frac{\partial}{\partial J}\left[V^{2}(J) \mathcal{J}_{\left[\frac{\omega_{0}}{\nu}\right]}^{2}(\xi) \frac{\partial F_{0}}{\partial J}\right] \\
& \frac{1}{W_{n}} \frac{\partial W_{n}}{\partial \theta}=\frac{i \pi \epsilon^{2} n}{2 \nu \sin \left(\pi \omega_{0} / \nu\right)} \mathcal{J}_{-\frac{\omega_{0}}{\nu}}^{2}(\xi) \frac{\mathrm{d}}{\mathrm{d} J}\left(V \frac{\mathrm{d} V}{\mathrm{~d} J}\right)+\frac{\pi \epsilon^{2} n}{2 \nu} \mathcal{J}_{\left[\frac{\omega_{0}}{\nu}\right]}^{2}(\xi) \frac{\mathrm{d}}{\mathrm{d} J}\left(V \frac{\mathrm{d} V}{\mathrm{~d} J}\right) .
\end{aligned}
$$

Equation (5.7) suggests that the amplitudes $W_{n}$ of the angular modes $G_{n}$ exhibit exponential growth with an increment

$\Gamma=\frac{\pi \epsilon^{2}}{2 \nu} \mathcal{J}_{\left[\frac{\omega_{0}}{\nu}\right]}^{2}(\xi) \frac{\mathrm{d}}{\mathrm{d} J}\left(V \frac{\mathrm{d} V}{\mathrm{~d} J}\right)$.

Equation (5.6) is a Fokker-Planck equation for the angle-independent part of the phase space density with a diffusion coefficient

$\mathcal{D}(J)=\frac{\pi \epsilon^{2}}{2 \nu} V^{2}(J) \mathcal{J}_{\left[\frac{\omega_{0}}{\nu}\right]}^{2}(\xi)$

In Figures 13 the reduced diffusion coefficient

$\mathcal{D}^{(R)}\left(J, \frac{\Xi}{\nu}\right)=\frac{2 \Xi}{\pi \epsilon^{2} V^{2}(J)} \mathcal{D}(J)$

has been plotted as a function of the ratio between the amplitude and the frequency of the modulation. Three typical regimes corresponding to different values of $\lambda / \Xi$ used as a control parameter have been chosen. In the first one depicted in Figure 1 we have taken the resonance detuning twice as large as the amplitude of the modulation $(\lambda=2 \Xi)$. In this case there is no crossing of the main resonance described by the Hamiltonian (5.1) and the diffusion coefficient decreases very rapidly after reaching its maximum value at $\xi=0.25$. The cases of periodic resonance crossings for $\lambda=\Xi$ and $\lambda=\Xi / 2$ are shown in Figure 2 and Figure 3, respectively.

\section{Concluding Remarks}

In the present paper we apply the Renormalization Group method to the reduction of non integrable multi-dimensional Hamiltonian systems. The notion of reduction is used here in the sense of slow, long-time behavior, that survives appropriate averaging and/or factorizing of rapidly oscillating contributions to the dynamics of the system.

As a result of the investigation performed we have derived evolution equations for the slowly varying part of the angle-averaged phase space density, and for the amplitudes of 
the angular modes. It has been shown that these equations are the Renormalization Group equations.

The case of a one-dimensional isolated nonlinear resonance with a resonant phase (or linear unperturbed tune) subjected to periodic modulation has been studied in detail. The coefficient of modulational diffusion, as well as the exponential growth increment of the amplitudes of angular modes have been obtained in explicit form.

\section{Acknowledgments}

It is a pleasure to thank E. Startsev for many helpful discussions concerning the subject of the present paper. I am indebted to N. Goldenfeld for careful reading of the manuscript,

and for making valuable suggestions. This work was supported by the U.S. Department of Energy. 


\section{REFERENCES}

1. B.V. Chirikov, Physics Reports, 52, 263 (1979).

2. A.J. Lichtenberg and M.A. Lieberman, Regular and Stochastic Motion, Springer-Verlag, Berlin 1983.

3. S. Chattopadhyay et al. editors, Nonlinear Dynamics in Particle Accelerators: Theory and Experiments, Arcidosso, Italy, 1994, AIP Conf. Proceedings 344, New York 1995.

4. S. Chattopadhyay et al. editors, Nonlinear and Collective Phenomena in Beam Physics, Arcidosso, Italy, 1996, AIP Conf. Proceedings 395, New York 1997.

5. V.I. Arnold and A. Avez, Ergodic Problems of Classical Mechanics, Benjamin, New York 1968.

6. R.W. Zwanzig, Lectures in Theoretical Physics, Vol. III, W.E. Brittin editor, John Wiley, New York 1961 (pp. 106-141).

7. L.-Y. Chen, N. Goldenfeld and Y. Oono Physical Review E, 54, 376 (1996).

8. K. Nozaki and Y. Oono Physical Review E, 63, 046101-1 (2001).

9. E.D. Courant and H.S. Snyder Annals of Physics, 3, 1 (1958).

10. A. Sudbery, Quantum Mechanics and the Particles of Nature, Cambridge University Press, Cambridge 1986. 


\section{FIGURES}

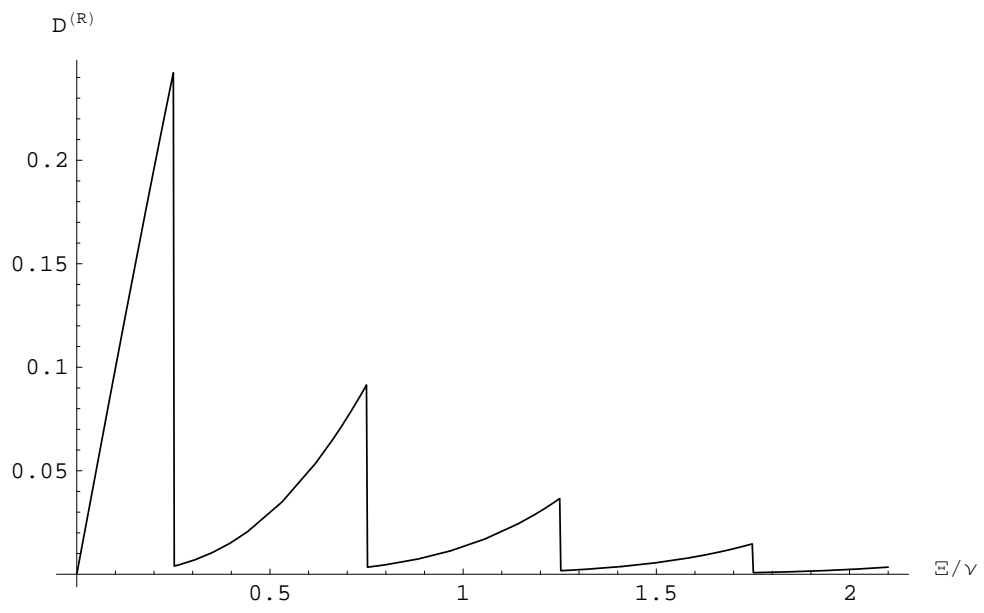

Fig. 1. Reduced diffusion coefficient $\mathcal{D}^{(R)}$ as a function of the ratio $\xi=\Xi / \nu$ for $\lambda=2 \Xi$. 


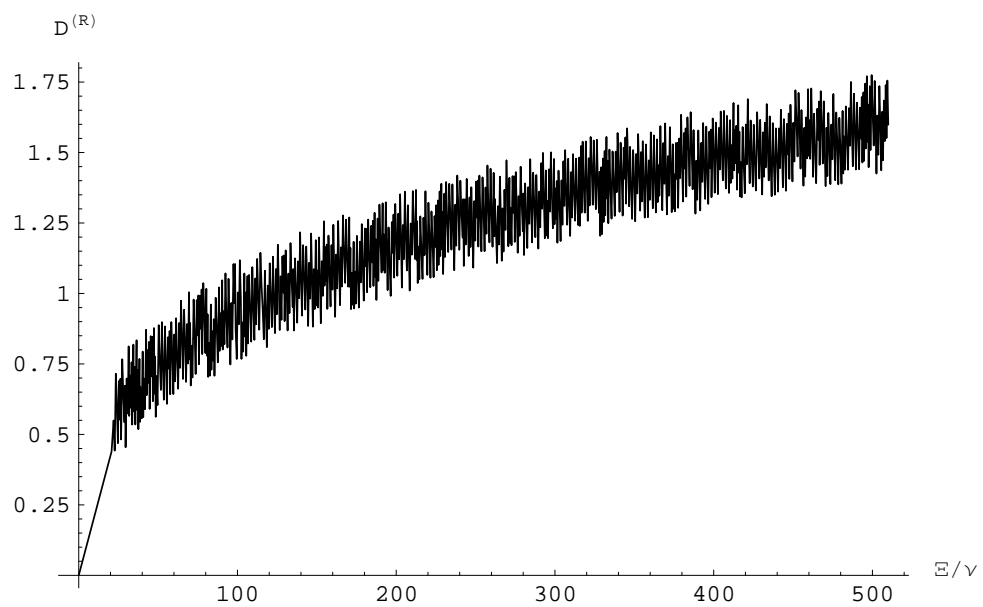

Fig. 2. Reduced diffusion coefficient $\mathcal{D}^{(R)}$ as a function of the ratio $\xi=\Xi / \nu$ for $\lambda=\Xi$. 


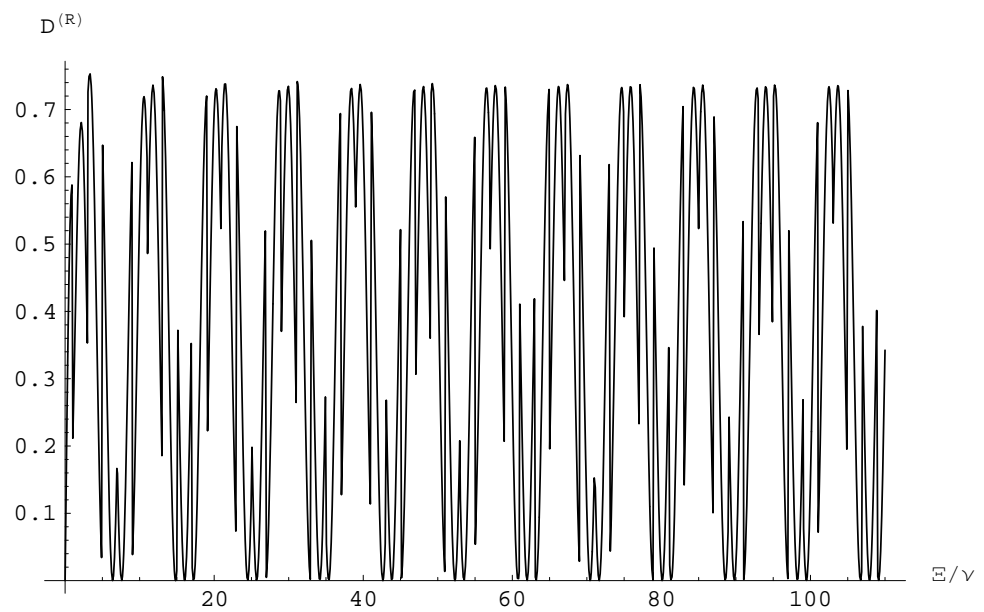

Fig. 3. Reduced diffusion coefficient $\mathcal{D}^{(R)}$ as a function of the ratio $\xi=\Xi / \nu$ for $\lambda=\Xi / 2$.. 